THE KURUME MEDICAL JOURNAL Vol. 1, No. 2, 1954

\title{
ÜBER DIE ARTERIOVENÖSE ANASTOMOSE IM THYMUS DER KATZE.
}

\author{
MASAHIRÔ MURAKAMI \\ Institut für Anatomie,* Medizinischen Fakultät, Universität \\ zu Kurume, Japan.
}

\section{EINLEITUNG}

Über die arteriovenösen Anastomosen in der Thymus bei Menschen, Hunden, Katzen und Ratten, berichtete Monroy (1940) folgendermassen, dass die arteriovenösen Anastomosen durch kurze Stämmchen verkörpert werden und sie nicht nur aus Arterien und Venen, sondern auch aus Arteriolen und Venulen bestehen.

Tondo (1941) beobachtete die gleichartigen Anastomosen in der Thymus von Katzen und Meerschweinchen, und demnächst nachwies Märk (1941) bei Menschen, und dann Teichmann $(1940,1942)$ bei Coronella und Tropidonus, kleine mit epitheloiden Zellen versehenen Arterien im Thymus. Nach den Meinungen Yamadas und Hosakas (1954) entwickeln sich die arteriovenösen Anastomosen von Menschen in der Grenzzone $z$ wischen der Rinde und dem Mark des Thymus und werden sie von retikulären Bindegeweben umgeschlossen, in deren Maschen zweiartige epitheloiden Zellen (die eine kleine, die andere grosse) vorhanden sind.

Ich habe die kleinen Blutgefässe in der interlobulären Bindegeweben des Thymus von der Katze untersucht und die einigen interessanten Resultate gewonnen.

\section{MATERIAL UND METHODE}

Als Untersuchungsmaterial dienten die Thymus von 4 Katzen (einem Fötus von $15 \mathrm{~mm} \times 50 \mathrm{~mm}$, einem neugeborene, 2 Kätzchen).

Nach der Einbettung in Zelloindin wurde das Material in $30 \mu$ Serienschnitte zerlegt, und dann mit Hämatoylin-Eosin gefärbt.

\section{EINIGE BEFUNDE}

a) Arteriovenöse Anastomose.

In der interlobulären Brindegeweben haben sich zwei arteriovenöse Anastomosen befunden. Die eine Anastomose besteht aus einer sigmoidförmig gewundenen

* Prof. Dr. Y. TAKeshige, M.D. 
Arterie (24 $\mu$ im Diameter) und einer Vene (114 $\times 57 \mu$ im Diameter), in der Tunica media der zuführenden Arterie liegen einige Zellen mit hellem Plasma, die mir epitheloide Zellen nach Schumacher erscheinen (Fig. 1).

Die andere hat die gestreckte Arterie (18 $\mu$ im Diameter) und die Vene $(24 \mu$ im Diameter). Diese zuführende Arterie, die den Kaliber an der Einmündengsstelle in die Vene abnimmt, besitzt keine epitheloiden Zellen (Fig. 2).

Ausserdem habe ich in dem Bindegewebe $z$ wischen der Trachea und dem Thymus eine arteriovenöse Anastomose gefunden, deren Arterie etwas gerade Verlauf zeigt, aber mit den epitheloiden Zellen versieht, wahrscheinlich wird diese Anastomose die Übergangsform der oben erwähnten beiden Anastomosen sein (Fig. 3).

Max Clara (1927) und Schumacher (1938) beschrieben wie folgend, dass in den arteriovenösen Anastomosen die eine vielfach gewundene Gruppe und die andere geradig verlaufende Gruppe vorhanden sind, jene in ihren Wandung die epitheloiden Zellen ausbilder, aber diese nicht. Hinsichtlich meiner Resultaten mögen die Meinungen von Max Clara und Schumacher auf die Thymus der Katzen anwendbar sein.

\section{b) Klappen der Venulen.}

Die Venenklappen befinden sich gewöhnlich an den Venen über 2-3 mm. im Diameter, aber die viszeralen Venen sind meist frei von Klappen.

Miyata (1953) bewies die Klappen der Venulen in der Zunge und Schilddrüse von Hund und Katze, auch habe ich in den interlobulären Bindegeweben der Thymus bei Katzen die Klappen zahlreich an den Venulen beobachtet, die den Durchmesser von 30 bis $230 \mu$ haben. Die meisten Klappen sind die bikuspidalen Taschenklappen und stehen nahe an den Einmündungsstellen seitlicher Venenäste (Fig. 4, $5,6)$.

\section{c) Ganglienzellen.}

In Bezug auf die Nervenzellen im Thymus wurde das sympathische Element nur von Terni (1929) bei Sauropsiden nachgewiesen, das, wie Terni sagt, die interstitiellen Zellen ebensogleich ist. Es scheint mir, dass die Nervenzellen im Thymus bei Säugetieren bisher von niemand erwiesen worden sind. Bei einem Kätzchen habe ich ein Geflecht $(78 \times 61 \mu$ im Diameter $)$ der Ganglienzellen im interlobulären Bindegewebe in der Nähe der dorsalen Fläche des Thymns und eine Ganglienzelle $(18 \times 10 \mu \mathrm{im}$ Diameter) in der Stelle gefunden, wo dasselbe Bindegewebe in das Innere des Thymus weiter eindringt (Fig. 7, 8). 


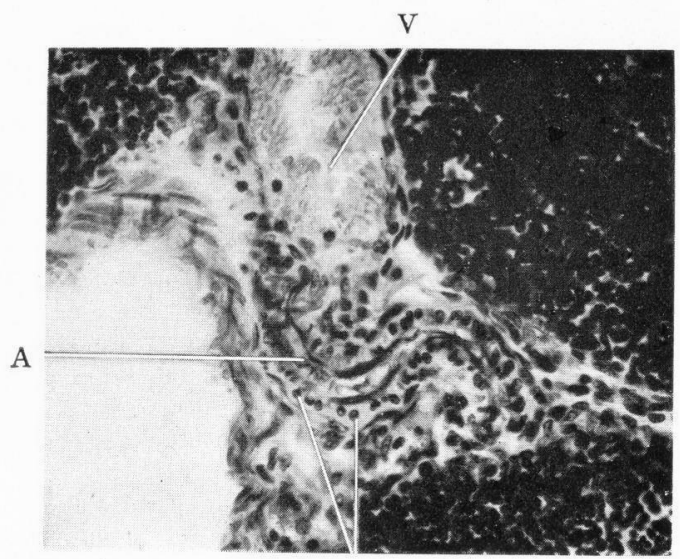

Ep

Fig. 1. Arteriovenöse Anastomose.
A : Arterie
$\mathrm{V}$ : Venule.
Ep : Epitheloide Zelle.
Vergr. 300 fach.

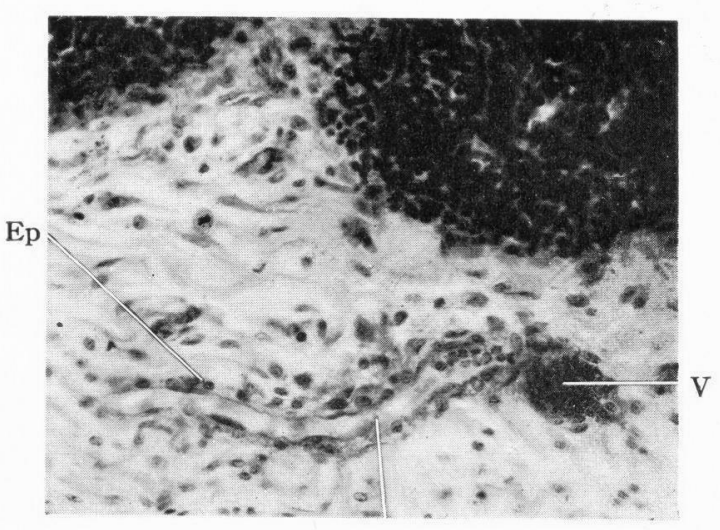

A

Fig. 3. Arteriovenöse Anastomose.
A : Arterie
$\mathrm{V}$ : Venule.
Ep: Epitheloide Zelle.
Vergr. 330 fach.

A

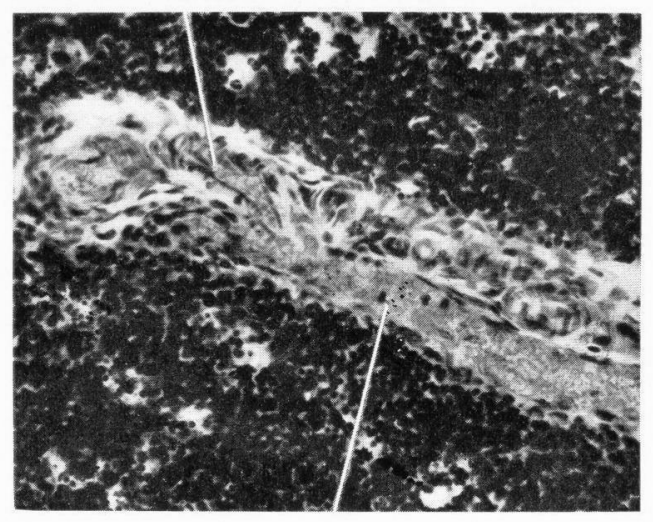

$\mathrm{V}$

Fig. 2. Arteriovenöse Anastomose.
A : Arterie.
$\mathrm{V}$ : Venule.
Ep: Epitheloide Zelle.
Vergr. 330 fach.

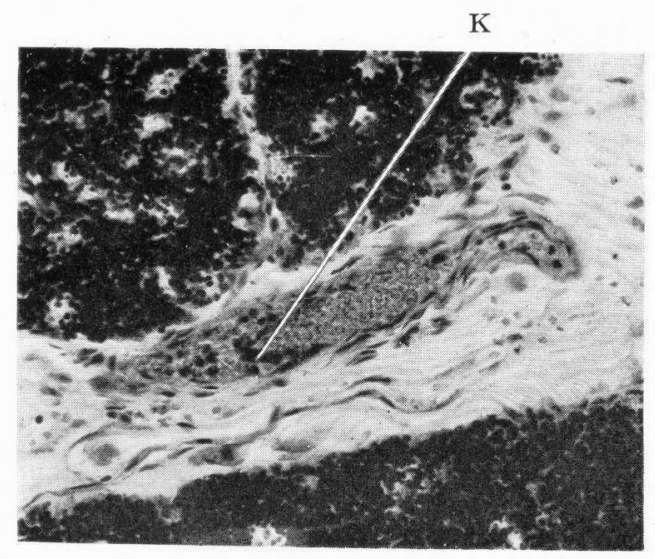

Fig. 4. Längsschnitt der Venule. (30 $\mu$ im Diameter).

K : Klappe.

Vergr. 270 fach. 
K

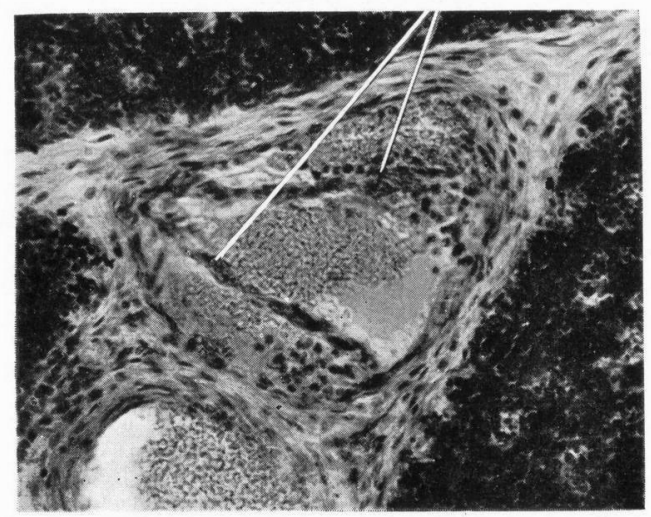

Fig. 5. Querschnitt der Venule.

(200 $\mu$ im Diameter).

$\mathrm{K}$ : Klappe.

Vergr. 260 fach.
K

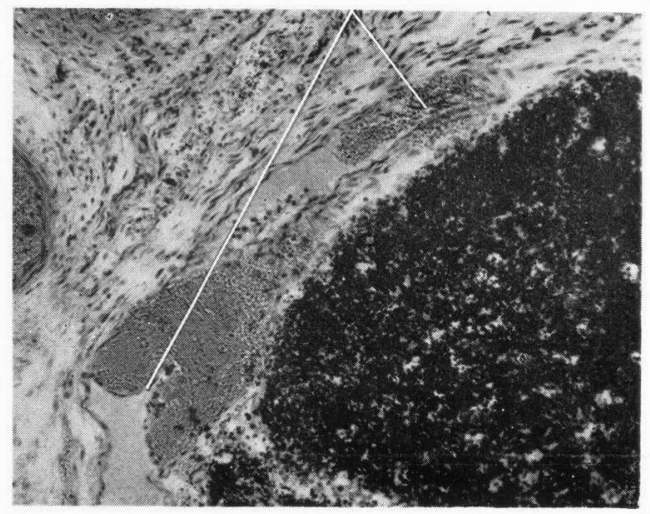

Fig. 6. Längsschnitt der Venulen an den Ein:nündungsstellen.

K : Klappe.

Vergr. 134 fach.

$\mathrm{G} z$

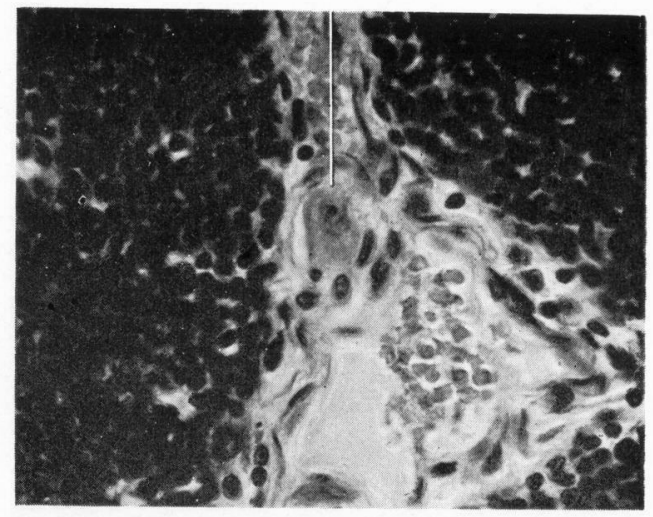

Fig. 8. Ganglienzelle.

G7: Ganglienzelle.

Vergr. 600 fach.

Vergr. 430 fach. 


\section{ZUSAMMENFASSUNG}

In der vorliegenden Arbeit habe ich bei vier Katzen hauptsächlich die arteriovenösen Anastomosen in interlobulären Bindegeweben der Thymus untersucht. Die wichtigen Resultate werden im folgend zusammenfassend angeführt.

1) In den interlobulären Bindegewebe der Thymus bei Katzen sind auch die arteriovenösen Anastomosen in den zweierlei Arten von M. Clara und Schumacher zu unterscheiden.

2) Die Klappen werden zahlreich in der Venulen des Thymus erwiesen.

3) Die Ganglienzellen befinden sich im interlobulären Bindegewebe des Thymus bei einem Kätzchen.

(Zum Beschluss dieser 'Arbeit stelle ich meinen herzlichsten Dank dem Prof. Dr. Y. Takeshige für seine Leitung und Revision, auch danke ich verstorbenem Herrn A. Tomita für seine Hilfe bei Verfertigung der Präparate.)

\section{LITERATURVERZEICHNIS}

1) Bargimann, W.: Der Thymus. Handbuch der mikroskopischen Anatomie des Menschen von Möllendorff., Bd. VI. Theil. 4. 1943.

2) Benninghoff, A.: Die Venenklappen. Handbuch der mikroskopischen Anatomie des Menschen von Möllendorff., Bd. VI. Theil 1. 1930.

3) Clara, M.: Die arteriovenösen Anastomosen. Leipzig. 1939.

4) Hammer, J. A.: Konstitutionsanatomosche Studien über die Neurotisierung des Menschenembryos. IV. Über die Innervationsverhältnisse der Inkretorgane und der Thymus bis in den 4. Fötalmonat. Z. mikrosk. -anat. Forsch., 38. 1935.

5) Kosтowizcкi, M.: Untersuchungen über Nervenendigungen in dem Thymus menschlicher Feten. Anat. Anz., 80. 1935.

6) Mixata, T.: Studies in arteriovenous anastomoses. Kyuschu Memoris of Medical Sciences. Vol. 3, No. 3. 1953.

7) Schumacher, S. : Über die Bedeutung der artəriovenösen Anastomosen und der epitheloiden Muskelzellen. Z. mikrosk. -anat. Forsch., Bd. 43.1938.

8) Ternt, T.: Ricerche istologische sull' innervazione del timo dei Sauropsidi. Z. Zellforsch. mikrosk. Anat., Bd. 9. 1929.

9) Uвока, W.: Über die Klappen der kleinen Venen in den Eingeweiden des Menschen. Nagoya. J. med. sci., 8. 1. 1934.

10) Yamada, H. and Hosaka, T.: Neurological and angiological studies on the medulla of human thymus. The bulletin of Tokyo medical and dental university. No. 1. 1954, 